\title{
Estudo tomográfico do posicionamento femoral em reconstruções, por técnica transtibial, do LCA
}

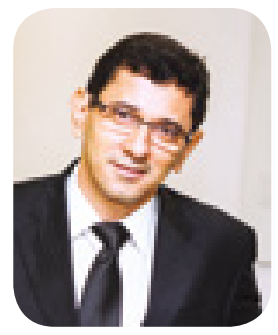

\author{
Robson Rocha da Silva ${ }^{1}$, Marcos Almeida Matos ${ }^{1}$, Valonie Carlos Neves \\ Brasileiro Costa ${ }^{1}$,Vitor Hugo Abreu Azevedo de Morais ${ }^{1}$, Luiz Eduardo \\ Lago de Castro
}

\begin{abstract}
Artigo Original: Tomographic Study of Femoral Positioning in Anterior Cruciate Ligament Reconstruction Using the Transtibial Technique. Autores: Robson Rocha da Silva, Marcos Almeida Matos, Valonie Carlos Neves Brasileiro Costa, Vitor Hugo Abreu Azevedo de Morais and Luiz Eduardo Lago de Castro. Publicado em : Knee Surg Relat Res. 2017 Sep; 29 (3):
\end{abstract} 195-202.

\section{INTRODUÇÃO}

A reconstrução do ligamento cruzado anterior (LCA) é uma das cirurgias ortopédicas mais realizadas atualmente. Nas últimas duas décadas, a técnica de reconstrução transtibial, que consiste na confecção do túnel femoral pelo túnel tibial, consagrou-se como um método rápido, reprodutível e de fácil execução. Rapidamente alcançou ampla aceitação, sendo largamente utilizada, com a publicação de bons resultados atribuídos à mesma.

Contudo, estudos recentes têm melhorado o conhecimento a respeito da anatomia do LCA, revelando que técnicas convencionais, tais como a transtibial, não proporcionam a replicação das inserções ligamentares originais. Em especial na técnica TT, o túnel tibial determina o túnel femoral, o que muitas vezes resulta numa orientação vertical do enxerto. Enxertos verticalizados exibem um controle rotacional inferior que resultam em artrite secundária. Nos últimos anos, a colocação do túnel mais anatômico tem sido comprovada para fornecer dados biomecânicos e cadavérico mais superior. Desta forma, as mudanças nos paradigmas da cirurgia com respeito ao posicionamento dos túneis numa posição mais anatômica têm levado a novas técnicas de reconstrução, que buscam restaurar, de forma mais precisa, a biomecânica articular, aproximando o joelho da sua originalidade. Além da proposição de novas técnicas, alterações têm sido sugeridas à técnica transtibial, no intuito de melhorar o posicionamento do túnel femoral ${ }^{3}$. Essas modificações baseiam-se na identificação apurada das inserções ligamentares e na rotação do guia femoral na parte posterior do côndilo femoral lateral, colocando o fio guia o mais próximo possível do ponto anatômico.

\section{MÉTODO}

Tratou-se de uma pesquisa exploratória em uma série de pacientes provenientes de um hospital de referência em cirurgia do joelho, escolhidos através de técnica da amostragem não probabilística e sequencial, no período de abril de 2013 até novembro de 2014. Todos os procedimentos foram realizados pelo mesmo cirurgião e submetidos a uma reconstrução não anatômica da LCA com autotransplante de isquiotibiais de feixe único, pela mesma técnica de reconstrução transtibial modificada (MTT).

Foram utilizados os seguintes critérios de inclusão: pacientes com idade entre 18 e 60 anos, reconstrução primária do LCA (lesão isolada do LCA) sem lesões ligamentares associadas. Os critérios de exclusão foram: alterações tomográficas na femoral lateral (artrose, displasia, cirurgia de revascularização, sequelas de fraturas ou infecção), interrupção no acompanhamento ou não realização da TC 3D após a cirurgia.

\section{RESULTADO}

$\mathrm{Na}$ comparação com os dados dos estudos tomados como referência para a reconstrução transtibial ${ }^{2}$, houve maior aproximação do ponto ideal com diferença estatisticamente significativa no plano vertical $(p<$ $0,005)$. Nas comparações com a média das coordenadas para a reconstrução anatômica transportal de fora para dentro e anatômica ideal descritas por Bird et. al. ${ }^{8}$ houve diferença estatística nos dois planos (tabelas 4 e 5). O posicionamento médio do túnel femoral, obtido neste estudo através da tomografia computadorizada em 3D, assim como o da técnica transtibial publicada na literatura, foi comparado ao posicionamento ideal e encontra-se na figura 3. 
A comparação do posicionamento deste estudo com o posicionamento das técnicas anatômicas transportal de fora para dentro e anatômica ideal encontra-se nas figuras 4 e 5 .

\section{DISCUSSÃO}

Em comparação com as coordenadas, publicadas na literatura, em reconstrução transportal convencional, nossos resultados apresentaram diferença estatística nos dois planos; no entanto, apenas no plano paralelo à linha de Blumensaat, essa diferença favoreceu à reconstrução transportal que atingiu, em média, o ponto anatômico de referência. No plano transversal, a diferença encontrada foi favorável aos nossos achados, inferindo que o posicionamento femoral obtido em nossas cirurgias se aproximou mais do posicionamento ideal anatômico. Bird et al., em estudo comparando o posicionamento do túnel femoral em técnica transportal convencional com uma técnica anatômica modificada, observaram que a técnica convencional produziu um túnel femoral mais próximo à linha de Blumenssat no plano transverso e atribuiu esse achado à dificuldade em identificar por via artroscópica, de forma precisa, os pontos de referência do footprint femoral.

$\mathrm{Na}$ comparação que realizamos dos nossos dados com a média das coordenadas de estudos de referência utilizando a técnica transtibial, observamos que, no plano paralelo à linha de Blumensaat, o túnel femoral manteve-se significativamente anterior ao sítio ideal. No entanto, no plano perpendicular à linha de Blumen- saat, alcançamos um melhor posicionamento na comparação com outros estudos com a técnica transtibial.

A comparação realizada neste estudo confirma que a técnica transtibial pode ser melhorada, porém não atinge sistematicamente o ponto anatômico. A técnica anatômica de fora para dentro, nessa comparação, aproximou-se mais do posicionamento ideal.

\section{CONCLUSÃO}

A partir desses dados podemos concluir que as médias obtidas em nosso estudo atingiram um melhor posicionamento do que as encontradas em técnicas transtibiais tradicionais, porém não atingindo o posicionamento ideal descrito na literatura. Podemos atribuir essa melhora do posicionamento à manobra rotacional aplicada ao guia femoral combinada ao melhor direcionamento do túnel tibial, a fim de obter um túnel femoral mais próximo da anatomia original.

Tabela 1. Comparação das coordenadas da tomografia computadorizada do presente estudo com as coordenadas de estudos de referência realizados com técnica transtibial.

\begin{tabular}{llllc}
\hline \hline & Amostra* $^{*}$ & Bird et al. $^{*}$ & Valor de P & IC 95\% \\
$\begin{array}{l}\text { TC Coord } \\
\text { Horizontal }\end{array}$ & $37,5 \pm 5,7$ & 30,0 & $<, 001$ & 4,5 a 10,4 \\
$\begin{array}{l}\text { TC Coord } \\
\text { Vertical }\end{array}$ & $23,5 \pm 6,7$ & 17,0 &, 001 & 3,0 a9,7 \\
\hline \hline
\end{tabular}

*Os valores são apresentados em média e desvio padrão.
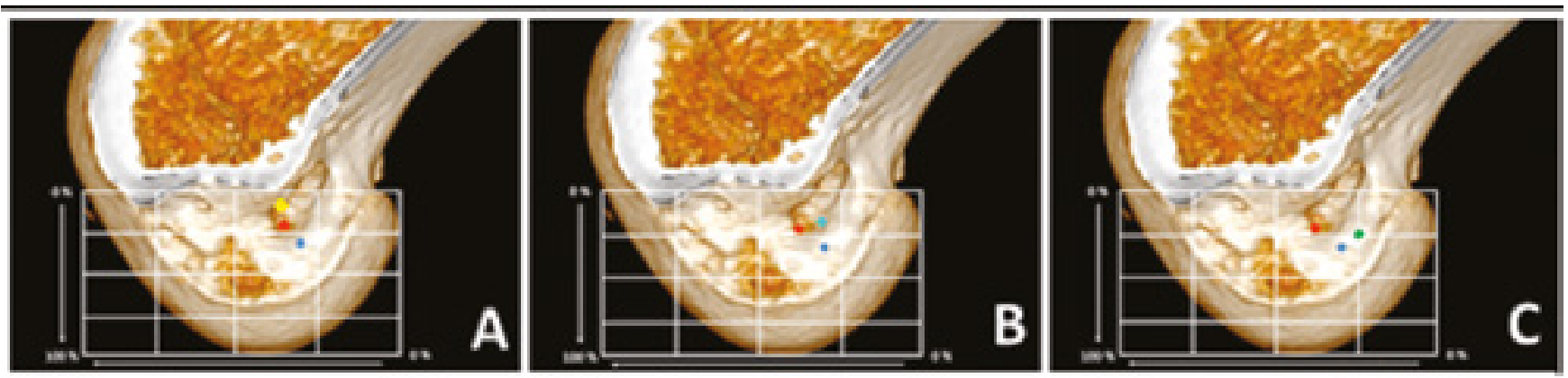

Figura 1. In yellow- Mean value of positions obtained in the reference studyfor the femoral tunnel, performed by TT technique, (Kopf et al.2) (37.2x11.3). In red- Mean value of positions of the present study (37.5x23.5). In blue- Ideal anatomic position, described in the literature, Bird et al.8, (28.0x35.0). 3B-In light blue- Mean values of femoral tunnel positions, performed by transportal technique, Bird et al.8,(30.0x17.0). In red- Mean value of positions of the present study (37.5x23.5).In blue-Ideal anatomic position, described in the literature, Bird et al.8, (28.0x35.0).3CIn green- Mean values of femoral tunnel positions, performed byoutside-in technique, Ahn et al. (27.0, 24.7). In red- Mean value of positions of the present study $(37.5 \times 23.5)$.In blue- Ideal anatomic position, described in the literature, Bird et al.8, (28.0x35.0). 


\section{REFERÊNCIAS}

1- Buoncristiani AM, Tjoumakaris FP, Starman JS, Ferretti $\mathrm{M}$, Fu FH. Anatomic double-bundle anterior cruciate ligament reconstruction. Arthroscopy. 2006 Sep;22(9):1000-6.

2- Kopf S, Forsythe B, Wong AK, Tashman S, Anderst W, Irrgang JJ, Fu FH. Nonanatomic tunnel position in traditional transtibial single-bundle anterior cruciate ligament reconstruction evaluated by three-dimensional computed tomography.J Bone Joint Surg Am. 2010 Jun;92(6):1427-31.

3-Ahn JH, Jeong HJ, Ko Chung-Suk, Ko TS, Kim JH. Three-Dimensional reconstruction computed tomography evaluation of tunnel location during single-bundle anterior cruciate ligament reconstruction: A comparison of transtibial and 2-incision tibialtunel-independent techniques.

Clinics in Orthopedic Surgery. 2013;5:26-35.

4-Kopf S, Forsythe B, Wong AK, Tashman S, Irrgang JJ, Fu FH. Transtibial ACL reconstruction technique fails to position drill tunnels anatomically in vivo 3D CT study. Knee Surg Sports TraumatolArthrosc. 2012;20:2200-7.

5- Hosseini A, Lodhia P, Van de Velde SK, Asnis PD, Zarins B, Gill TJ, Li G. Tunnel position and graft orientation in failed anterior cruciate ligament reconstruction: a clinical and imaging analysis. IntOrthop. 2012;36(4):84552.

6- Carson EW, Anisko EM, Restrepo C, Panariello RA, O'Brien SJ, Warren RF. Revision anterior cruciate ligament reconstruction: etiology of failures and clinical results. J Knee Surg. 2004;17(3):127-32.

7- Dienst M, Burks RT, GreisPE. Anatomy and biomechanics of the anterior cruciate ligament.OrthopClin North Am. 2002 Oct;33(4):605-20.

8- Bird JH, Carmont MR, Dhillon M, Smith N, Brown C, Thompson P, Spalding T. Validation of a new technique to determine midbundle femoral tunnel position in anterior cruciate ligament reconstruction using 3-dimensional computed tomography analysis. Arthroscopy. 2011 Sep;27(9):1259-67.

9- Basdekis G, Christel P, Anne F. Validation of the position of the femoral tunnels in anatomic double-bundle ACL reconstruction with 3-D CT scan. Knee Surg Sports TraumatolArthrosc. 2009 Sep;17(9):1089-94.

10-Bernard M, Hertel P. Intraoperative and postoperative insertion control of anterior cruciate ligament-plasty. A radiologic measuring method (quadrant method). Unfallchirurg. 1996 May;99(5):332-40.

11-Asagumo H, Kimura M, Kobayashi Y, Taki M, Takagishi $\mathrm{K}$. Anatomic reconstruction of the anterior cruciate ligament using double-bundle hamstring tendons: surgical techniques, clinical outcomes, and complications. Ar- throscopy. 2007 Jun;23(6):602-9.

12- Heming JF, Rand J, Steiner ME. Anatomical limitations of transtibial drilling in anterior cruciate ligament reconstruction.Am J Sports Med. 2007 Oct;35(10):1708-15

13- Getelman $\mathrm{MH}$, Friedman MJ. Revision anterior cruciate ligament reconstruction surgery. J Am AcadOrthop Surg. 1999 May-Jun;7(3):189-98.

14- Piasecki DP, Bach BR Jr, Espinoza Orias AA, Verma NN. Anterior cruciate ligament reconstruction: can anatomic femoral placement be achieved with a transtibial technique? Am J Sports Med. 2011 Jun;39(6):1306-15.

15- Steiner ME, Battaglia TC, Heming JF, Rand JD, Festa A, Baria M. Independent drilling outperforms conventional transtibial drilling in anterior cruciate ligament reconstruction. Am J Sports Med. 2009 Oct;37(10):1912-9.

16 - Cohen SB, Fu FH. Three-portal technique for anterior cruciate ligament reconstruction: use of a central medial portal. Arthroscopy. 2007 Mar;23(3):325.e1-5.

17- Fernandes TL, Martins NMMF, Watai FA, Neto CA, Pedrinelli A, Hernandes AJ. Tomografia computadorizada 3D para mensuração do posicionamento femoral na reconstrução do LCA. Acta Ortopédica Br. 2015;23(1): 11-5.

18- Bedi A, Musahl V, Steuber V, Kendoff D, Choi $D$, Allen AA, Pearle AD, Altchek DW. Transtibial versus anteromedial portal reaming in anterior cruciate ligament reconstruction: an anatomic and biomechanical evaluation of surgical technique. Arthroscopy. 2011 Mar;27(3):380-90.

19- Dargel J, Schmidt-Wiethoff R, Fischer S, Mader K, Koebke J, Schneider T. Femoral bone tunnel placement using the transtibial tunnel or the anteromedial portal in ACL reconstruction: a radiographic evaluation. Knee Surg Sports TraumatolArthrosc. 2009 Mar;17(3):220-7.

20- Pinczewski LA, Salmon LJ, Jackson WF, von Bormann RB, Haslam PG, Tashiro S. Radiological landmarks for placement of the tunnels in single-bundle reconstruction of the anterior cruciate ligament. J Bone Joint SurgBr. 2008 Feb;90(2):172-9.

21- Howell SM. Deutsch ML. Comparison of endoscopic and two-incision techniques for reconstructing a torn anterior cruciate ligament using hamstring tendons. Arthroscopy. 1999;15(6):594-606.

1- Serviço de Ortopedia do HSI

2- Serviço de Imagem do HSI

Endereço para correspondência:

robroc@superig.com.br 